\title{
Flow and Heat Transfer over an Exponential Stretching Sheet under the Effects of a Temperature Gradient Dependent Heat Sink and Thermal Radiation.
}

\author{
${ }^{1}$ J.K.Nagbhooshan, ${ }^{2}$ P.H.Veena, ${ }^{3}$ K.Rajagopal, ${ }^{4}$ V.K.Pravin \\ ${ }^{l}$ Department of Mathematics, ABPS, Dist. Gulbarga, Karnataka, INDIA \\ ${ }^{2}$ Department of Mathematics, Smt. V.G.College for Women Gulbarga, Karnataka, INDIA \\ ${ }^{3}$ Department of Mechanical Engineering, JNTUniversity, Hyderabad, A.P., INDIA \\ ${ }^{4}$ Department of Mechanical Engineering, P.D.A. College of Engg. Gulbarga, Karnataka, INDIA
}

\begin{abstract}
The problem of MHD flow and heat transfer in a Newtonian viscous incompressible fluid over a stretching sheet with temperature gradient dependent heat sink/source and radiation is investigated. The governing partial differential equations are converted into ordinary differential equations by similarity transformation technique. The effects of viscous dissipation, work due to deformation, thermal radiation are considered in the energy equation and the variations of dimensionless surface temperature as well as the heat transfer characteristics with various values of non-dimensional parameters like Prandtl number, suction parameter, radiation parameter, temperature gradient dependent heat sink parameter are graphed and tabulated. The heating process of the type $i$ ) the sheet with prescribed surface temperature (PST case) is studied.
\end{abstract}

Key words; MHD, viscous fluid, stretching sheet, radiation parameter, temperature gradient dependent heat sink,

\section{Introduction}

Boundary layer behavior over a continuous moving flat sheet finds wide applications in engineering and manufacturing process in industry. These include aerodynamics extrusion of plastic sheets, rolling and extrusion in manufacturing process, the cooling of an infinite metallic plate in a cool path boundary layer along a liquid film in condensation process, the boundary layer along the material handling conveyers etc.

Since the pioneering work of Sakiadis[19] presented studies on boundary layer flow adjacent to containing moving surface in the context of chemical engineering systems. McComack \& Crane[14] have provided comprehensive analysis on boundary layer flow including the flow caused by stretching of flat surface and between two surfaces under different physical situations. Since then many authors including Rajgopal et al [18], Wang [26], Thakar and Soundalgekar [24], Surmadevi et al [22], have studied the boundary layer flow caused by a stretching / continuously moving sheet for different thermo physical situation using variety of fluid models and boundary conditions.

Thermal radiation effect might play a significant role in controlling heat transfer phenomena in polymer processing industry. The quality of the final product depends to an extent on the heat controlling factors. In view of this Raptis and Perdikis [1] analysed visco-elastic flow and heat transfer past a semi-infinite porous plate having constant suction of the fluid in presence of thermal radiation. Viscous dissipation which must be taken into account in the heat transfer analysis of non-Newtonian fluid flow is excluded from this stuffy. Raptis [2] studied boundary layer flow and heat transfer of micro polar fluid past a continuously moving plate with viscous dissipation in the presence of radiation. Raptis [3] also investigated the visco-elastic fluid flow past a semi infinite plate taking into consideration of radiation using Rosseland approximation Brewester [12] when the free stream velocity and the temperature of the plate are not constant. However, this work does not deal with the situation when there would be a temperature dependent heat source/sink, viscous dissipation and suction/blowing through the porous boundary surface.

Kumari and Nath [11] studied radiation effect in a non-Darcy mixed convection flow over a solid surface immersed in a saturated porous medium using Rosseland approximation. Rafael [17] studied about visco-elastic fluid flow and heat transfer over a stretching sheet under the effects of a non uniform heat source, viscous dissipation and thermal radiation. Another effect which bears great importance on heat transfer in the viscous dissipation the determination of the temperature distribution when the internal friction is not negligible is of utmost significance in different industrial fields, such as chemical and food processing, oil exploitation and bio-engineering. Consequently, the effect of viscous dissipation is also included in the energy equation.

On the other hand, the effect of radiation on visco-elastic boundary layer flow and heat transfer problems can be quite significant at high operating temperature. In view of this, visco-elastic flow and heat 
Flow and heat transfer over an exponential stretching sheet under the effects of a temperature

transfer over a flat plate with constant suction, thermal radiation and without viscous dissipation were studied by Raptis and Perdikis [1]. Viscous dissipation and radiation were considered by Raptis [2] and the effect of radiation was also included in reference [3] and [4]. Very recently, researches in this field have been conducted by many investigators[21], however, the effects of work due to information on visco-elastic flows and heat transfer in the presence of radiation, viscous dissipation and uniform heat source/sink have not been studied in recent years.

Hong and Tien [9] investigated the thermal dissipation effects on free convection heat transfer in a porous medium bounded by a vertical plate. Angirasa et al [6] provided on an analysis on heat and mass transfer effects in internally satisfied porous medium for unsteady boundary layer flow. In the present century, a century of technological advancement, exploration of industries using latest technologies in extrusions in manufacturing processes and melt-spinning processes in taking place. In the industries the extradites is stretched into a filament when it is drawn format their dye and solidifies in the desired shape through a controlled cooling system. Then for many authors including. Thakar et al [23], Anjalidevi and Thiyagrajan [7], Khan et al [10] have analysed various problems on stretching sheet for different flow model.

It is known that thermal boundary layer equation with viscous dissipation term is non-homogeneous partial differential equation involving quadratic power of the velocity gradient. To seek a similarity solution of the thermal boundary layer equation, in case of linear stretching problem, we contemplate to deal with quadratic power law thermal boundary conditions for a general case of boundary heating of the type prescribed power law surface temperature of second degree (PST). Several closed form analytical solutions for the heat transfer characteristics are obtained in the form of confluent hypergeomtric function (Kummer's functions).

\section{Mathematical Formulation}

In certain coordinate system $(\mathrm{x}, \mathrm{y})$, consider two dimensional free convention steady laminar boundary layer flow of an incompressible, viscous fluid caused by a moving porous sheet embedded in porous medium in presence of a temperature gradient dependent heat sink. The porous sheet is subjected to a constant suction velocity normal to the wall. The axis is taken along wall in the direction of motion of the flow and y-axis perpendicular to it. Let the components of velocity be $\mathrm{u}$ and $\mathrm{v}$ along $\mathrm{x}$ and $\mathrm{y}-$ directions respectively. It is envisaged that the sheet issues from a thin slit at the origin $(0,0)$ and the speed at a point on the plate is proportional to its distance from the plate but the boundary layer approximation still hold. Under the above mentioned assumptions and the following Vafai and Tien's [25] model, the steady state boundary layer equations of mass and momentum are:

$$
\begin{aligned}
& \frac{\partial \mathrm{u}}{\partial \mathrm{x}}+\frac{\partial \mathrm{v}}{\partial \mathrm{y}}=0 \\
& u \frac{\partial u}{\partial x}+v \frac{\partial u}{\partial y}=v \frac{\partial^{2} u}{\partial y^{2}}-\frac{v}{k} u-\frac{\sigma B_{0}^{2}}{\rho} u
\end{aligned}
$$

The supplementary term in the momentum equation namely the Darcian body force term $-\frac{v}{k} u$ is linear interms of the $\mathrm{x}$-direction of the stretching motion.

The appropriate boundary conditions for the problem are:

$$
\begin{aligned}
& \mathrm{u}=\mathrm{bx}, \quad \mathrm{v}=-\mathrm{v}_{\mathrm{w}}, \quad \text { at } \quad \mathrm{y}=0 \\
& \mathrm{u}=0, \quad \text { as } \quad \mathrm{y} \quad \infty
\end{aligned}
$$

\section{Solution of Momentum Equation}

To solve equation (2), we postulate a solution to the velocities in $\mathrm{x}$ and $\mathrm{y}$ directions as follows:

$$
u=b x G^{\prime}(\eta), \quad v=-\sqrt{b v} G(\eta) \text { and } \eta=\sqrt{\frac{b}{v}} y
$$

by using $u$ and v, equation of continuity (1) is satisfied. Substituting equation (4) in equation (2), we obtain:

$$
G^{\prime \prime}(\eta)+G(\eta) G^{\prime \prime}(\eta)-G^{\prime 2}(\eta)-\frac{1}{\left(K_{2}+M\right)} G^{\prime}(\eta)=0
$$

By using (4), the boundary conditions(3) corresponding to the equation (2) reduces to:

$$
\begin{aligned}
& G^{\prime}(\eta)=1 \text {, } \\
& G(\eta)=S \\
& \text { at } \quad \eta=0 \\
& G^{\prime}(\eta)=0 \\
& \text { as } \quad \eta \rightarrow \infty
\end{aligned}
$$


Where $S=\frac{V_{w}}{\sqrt{v b}}$ is the suction parameter.

By using the boundary conditions (6) in equation (5) the solution is given us:

$G(\eta)=A+B e^{-E \eta}$

Where

$$
\begin{aligned}
A= & \frac{E^{2}-\left(K_{2}+M\right)^{-1}}{E}, \\
& B=-\frac{1}{E} \quad \& \quad E=\frac{1}{2}\left[S+\sqrt{S^{2}+4\left(1+\left(K_{2}+M\right)^{-1}\right)}\right]
\end{aligned}
$$

Hence the exact solution shown in (7) can be expressed as :

$G(\eta)=\frac{1}{E}\left[E^{2}-\left(K_{2}+M\right)^{-1}-e^{-E \eta}\right]$

Hence we have

$G^{\prime}(\eta)=e^{-E \eta}$

\section{Heat Transfer Analysis}

Energy equation by considering thermal radiation and temperature gradient dependent heat sink term is of the form

$u \frac{\partial T}{\partial x}+v \frac{\partial T}{\partial y}-\frac{K_{2}}{\rho C_{p}} \frac{\partial^{2} T}{\partial y^{2}}+\frac{1}{\rho C_{p}} \frac{\partial q_{r}}{\partial y}+Q^{\prime} \frac{\partial T}{\partial y}=0$

Where $\mathrm{k}_{2}$ is the thermal conductivity of the fluid, $\mathrm{T}$ is the fluid temperature, $\mathrm{q}_{\mathrm{r}}$ is the radiation heat flux and other quantities have usual meaning [10].

Using Rossel and approximation for radiation [12] we can write

$q_{r}=-\frac{4 \sigma}{3 k^{*}} \frac{\partial T^{4}}{\partial y}$

Here $\sigma$ is the Stefan - Boltzmann constant and $\mathrm{k}^{*}$ is the absorption coefficient. Further we assume that the temperature difference within the flow is such that $\mathrm{T}^{4}$ may be expanded in a Taylor series about $T_{\infty}$ and neglecting higher order terms we get

$T^{4} \equiv 4 T_{\infty}^{3} T-3 T_{\infty}^{4}$.

The supplementary term in the energy equation namely the temperature gradient dependent heat sink $Q^{\prime} \frac{\partial T}{\partial y}$ is a linear function of the temperature field.

Prescribed Surface Temperature (PST) case : In this case, the boundary conditions are

$$
\begin{array}{ll}
\mathrm{T}=\mathrm{T}_{\mathrm{w}}=\mathrm{T}_{\infty}+\mathrm{A}\left(\frac{x}{l}\right)^{2} & \text { at } \quad \mathrm{y}=0 \\
\mathrm{~T}=\mathrm{T}_{\infty} & \text { as } \mathrm{y} \rightarrow \infty
\end{array}
$$

where $l$ is the characteristic length.

To solve heat equation (10), we introduce the following non-dimensional quantity for the temperature variable $\mathrm{T}$ as

$\theta(\eta)=\frac{T-T_{\infty}}{T_{w}-T_{\infty}}$

using (4), (12) and (13) equation (10) reduce to

$\theta^{\prime \prime}+\frac{3 P_{r} N}{3 N+4}\left(1+Q^{*}\right) G \theta^{\prime}-\frac{3 P_{r} N}{3 N+4} 2 G^{\prime} \theta=0$

where 

$P_{r}=\frac{\mu C_{p}}{k_{2}}-\quad$ Prandtl number $\quad ; \quad N=\frac{\mu C_{p} K^{*}}{4 \sigma T_{\infty}^{3}}-\quad$ Radiation parameter for temperature as $\theta(\eta)=\frac{T-T_{\infty}}{T_{w}-T_{\infty}}$ and corresponding dimensionless boundary conditions are $\theta(\eta)=1 \quad$ at $\quad \eta=0$

$\theta(\eta) \rightarrow 0 \quad$ as $\quad \eta \rightarrow \infty$

To obtain the solution of equation (14), we introduce a new change of variable $\zeta$ defined as

$$
\zeta=\frac{-P_{r}\left(1+Q^{*}\right)}{E^{2}}\left(\frac{3 N}{3 N+4}\right) e^{-E \eta}
$$

Hence with the help of (12) and (16), equation (14) transforms to

$$
\zeta \theta^{\prime \prime}+\left[1-a_{0}-\zeta\right] \theta^{\prime}+\frac{2}{1+Q^{*}} \theta=0
$$

where $a_{0}=\left[\frac{P_{r}\left(1+Q^{*}\right)}{\alpha^{2}}-\frac{-P_{r}\left(1+Q^{*}\right)}{\alpha} \frac{V_{w}}{\sqrt{b v}}\right]\left(\frac{3 N}{3 N+4}\right) e^{-E \eta}$

and the corresponding boundary conditions converts to

$$
\theta\left(\frac{-P_{r}\left(1+Q^{*}\right)}{\alpha^{2}}-\frac{3 N}{3 N+4}\right)=0, \quad \theta(0)=1
$$

The solution of equation (17) under the boundary conditions (18) interms of confluent hypergeomtric function is obtained in terms of $\eta$ as

$$
\begin{gathered}
\theta(\eta)=e^{-E a_{0} \eta} \frac{M\left[a_{0}-S ; 1+a_{0} ;-K_{3} e^{-E \eta}\right]}{M\left[a_{0}-S ; 1+a_{0} ;-K_{3}\right]} \\
\text { where } S=\frac{2}{1+Q^{*}} \text { and } K_{3}=\left[\frac{-P_{r}\left(1+Q^{*}\right)}{E}\right]\left(\frac{3 N}{3 N+4}\right)
\end{gathered}
$$

\section{$\underline{\text { Skin Friction }}$}

The non-dimensional skin-friction $(\tau)$ at the wall $\eta=0$ is derived as

$$
\tau=\left(\frac{\partial u}{\partial \eta}\right)_{\eta=0}=b x G^{\prime \prime}(\eta)
$$

and obtained for different sets of values of permeability parameter $\mathrm{k}_{2}$, suction parameter $\mathrm{S}$ and stretching rate $\mathrm{bx}$ are tabulated in the following table 1 .

\begin{tabular}{|c|c|c|c|}
\hline $\mathbf{k}_{\mathbf{2}}$ & $\mathbf{S}$ & $\mathbf{b x}$ & $\boldsymbol{\tau}$ \\
\hline 10 & 0.5 & 1.0 & -1.32819 \\
\hline 20 & 0.5 & 1.0 & -1.30475 \\
\hline 40 & 0.5 & 1.0 & -1.29682 \\
\hline 30 & 1.0 & 1.0 & -1.56193 \\
\hline 30 & 1.5 & 1.0 & -2.13875 \\
\hline 30 & 0.5 & 1.5 & -1.89329 \\
\hline 30 & 0.5 & 2.0 & -2.76563 \\
\hline
\end{tabular}

Table 1. Skin-friction.

\section{Results and Discussion}

Results for the velocity profiles are depicted fig(1). By studying the velocity for various positions. From Fig(1a) and Fig (1b) it is seen that velocity decreases for increasing values of permeability parameter $k_{2}$ and magnetic parameter M. It is also observed that physically $\mathrm{k}_{2}$ expresses the pressure of porous matrix and quantities the hydraulic conductivity of the porous medium. The permeability acts as Darcy force term 
$\frac{-1}{k_{2}} G^{\prime}(\eta)=0$ for $k_{2} \rightarrow \infty$ in the transformed equation and serves to retard the momentum in the positive $\mathrm{x}$ direction.

Fig(1b), M express the pressure of magnetic field and the magnetic conductivity of the magnetic field. The magnetic parameter acts as magnetic force term and $\frac{-1}{M} G^{\prime}(\eta) \rightarrow 0$ for $M \rightarrow \infty$ in the equation and serves to retard the momentum in the positive $\mathrm{x}$-direction. Thus our results related to magnetic effect are correlated with the general conclusion arrived at by the studies of Thakar et al[23].

Fig(1c) shows the variation of velocity u i.e. $x$-direction longitudinal velocity versus $\eta$ for $S=0$ (non porous wall i.e. zero section). $S=0.5, S=1.0$ and $S=1.5$. It is observed that a steady decrease in $u$ and rise in suction parameter $\mathrm{S}$, with all profiles tending asymptotically to the horizontal axis. The longitudinal velocity $\mathrm{u}$ is observed to be a maximum in all cases at the wall i.e $\eta=0$. Schlichting[20] has shown that suction acts physically to increase the adherence to the wall of the hydrodynamic boundary layer, which in turn retards the flow in the longitudinal direction.

Fig(2a) shows the effect of permeability $\left(\mathrm{k}_{2}\right)$ on transverse velocity $\mathrm{G}(\eta)$ versus $\eta$. During the initial stage when the leading edge effect is not felt, the transverse velocity vis minimum and its maximum values always occur at the end of $\eta$ range and steadily rise from $\eta=0$ to $\eta=5$ and ultimately flattered towards the end of the range. The permeability of the porous structure $\mathrm{k}_{2}$ and suction $\mathrm{S}$ in the momentum equation (5) are inherently tied to the porous matrix and generally act in accordance with experimental finding (see Vafai \& Tien[25]). These results correlate well with the general conclusion arrived at by the studies of Ingham[18], Thakar et al[23].

Fig(2b) shows the effect of magnetic parameter M on transverse velocity $\mathrm{G}(\eta)$ versus $\eta$ physically $\mathrm{M}$ express the pressure of uniform magnetic field. Initially when magnetic effect is not felt at the edge, the velocity distribution is minimum and after wards it steadily increases and at the end of range of values of $\eta$ distribution is maximum and ultimately remains same till the last. The magnetic conductivity of the magnetic parameter $\mathrm{M}$ acts as magnetic force and as $\mathrm{M}$ increases which intern decreases the transverse velocity.

In fig (2c), shows the variation of transverse velocity i.e. $\mathrm{G}(\eta)$ versus $\eta$ for various values of suction parameter $\mathrm{S}=0.5,1.0,1.5$. It is observed from the figure that there is steady decrease in velocity accompanies a rise in $\mathrm{S}$ with all profiles tending asymptotically to the horizontal axis. The velocity is observed to be a maximum in all cases at the wall $\mathrm{u}$, when

$\eta=0$. Ajaykumar[5] has shown that suction acts physically to increase the adherence to the wall of the hydrodynamic boundary layer which intern retards flow longitudinal direction and velocity profiles are monotonically decreasing in nature.

Fig (3a) shows the variations in temperature $\theta(\eta)$ with $\eta$ for the effects of various combinations of $S$ and $Q^{*}$. Maximum temperature distribution is occurred at the curve 1 for which $S=0.5$ and $Q=0.5$. This physically means that an union of low suction with low wall temperature and weak heat sink. Further temperature distribution is decreasing for rise in values $S$ and $Q^{*}$ values. However the value of suction parameter S for curve II is higher than for the curve III and curve IV. This implies that S impinges relatively less effects on lowering the temperature in comparison to the values of $\mathrm{P}$ and $\mathrm{Q}^{*}$. Such a scenario implies that a stronger heat sink or greater wall temperature, parameter plays more dominant role for lowering the temperature.

Fig(3b) depicts the graph of variations in heat transfer with permeability parameter $\mathrm{k}_{2}$ illustrating the effects of $\mathrm{S}$ and $\mathrm{Q}^{*}$ values. As the heat sink parameter $\mathrm{Q}^{*}$ rises and for increasing values of permeability parameter $\mathrm{k}_{2}$ from curve I to curve IV, the temperature distribution is lowered. However for curve III, the $\mathrm{P}$ value is higher and for the curve II, the suction parameter $\mathrm{S}$ is higher which explains the fact that suction plays a more powerful role in reducing the temperature.

Fig(4a) is drawn for temperature profile for various values of Prandtl number $\mathrm{P}_{\mathrm{r}}$ and radiation parameter $\mathrm{N}$ when $\mathrm{V}_{\mathrm{w}}=-0.04$. This fig depicts the graphical representation of temperature profile for various values of Prandtl number $P_{r}$ and radiation parameter. The effect of increasing values of Prandtl number $P_{r}$ is to decrease temperature at a point in the flow field, as there would be a thinning of the thermal boundary layer, as a result of reduced thermal conductivity. The combined effect of increasing values of $\mathrm{P}_{\mathrm{r}}$ and $\mathrm{N}$ is to reduce temperature largely in the boundary layer flow region.

Fig(4b) is plotted for the same data sets as those of fig(4a) respectively except for $V_{w}=0.04$. In these cases temperature profiles follow similar pattern as in the case of fig (4a). However, the comparative study of fig(4a) and $(4 \mathrm{~b})$ reveals that the effect of blowing parameter $\mathrm{V}_{\mathrm{w}}=0.04$ is to increase the temperature throughout the boundary layer except on the wall where it attains unity in PST case. This is due to the fact that thermal boundary layer increases in the case of blowing. 

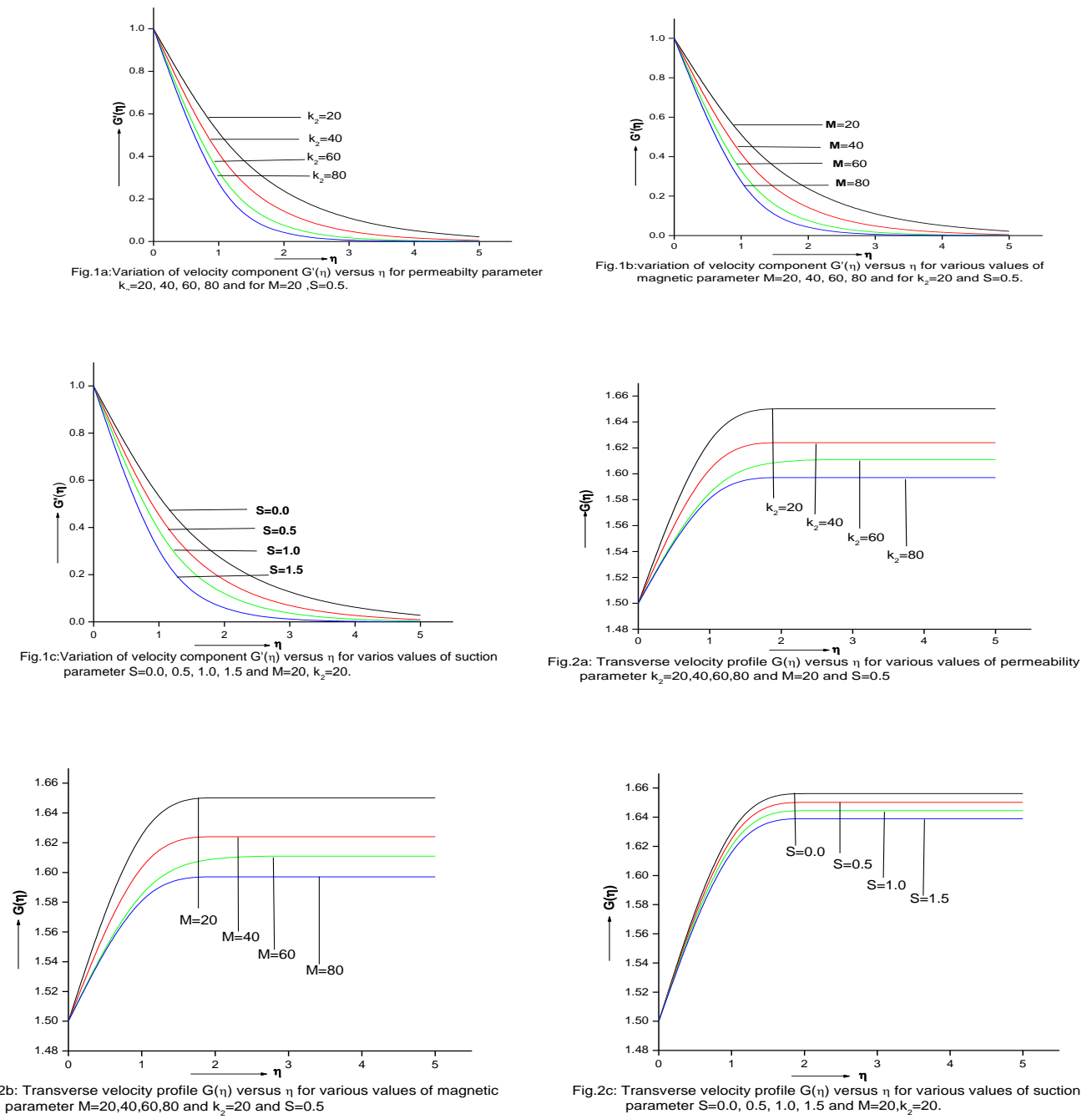

ig.2b: Transverse velocity profile $G(\eta)$ versus $\eta$ for various values of magnetic parameter $\mathrm{M}=20,40,60,80$ and $\mathrm{k}_{2}=20$ and $\mathrm{S}=0.5$

Fig.2c: Transverse velocity profile $\mathrm{G}(\eta)$ versus $\eta$ for various values of suction
parameter $\mathrm{S}=0.0,0.5,1.0,1.5$ and $\mathrm{M}=20, \mathrm{k}_{2}=20$.

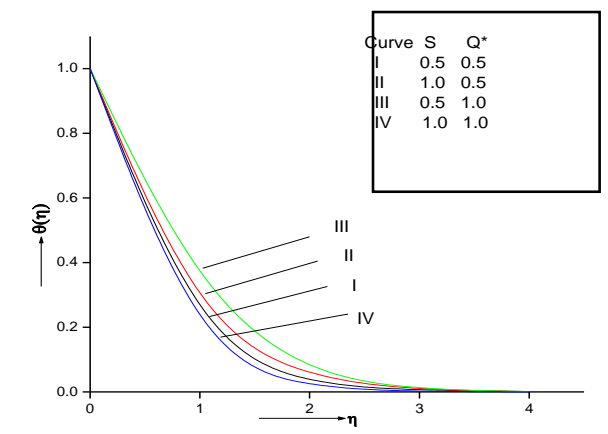

.3a:Dimensionless temperature profile versus $\eta$ for set of values of $S, Q^{*}$ and magnetic parameter $M=20,40,60,80$ permeability parameter $k_{2}=20$ in PST case.

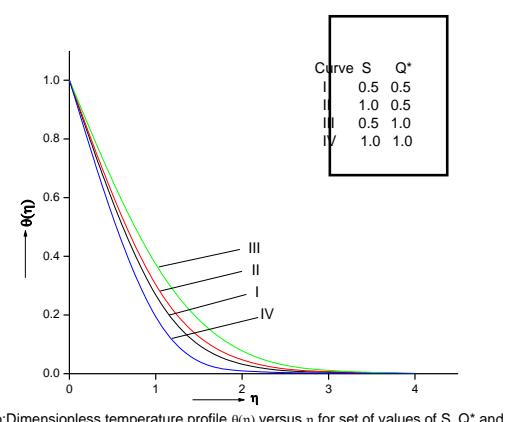

:ig.3b:Dimensionless temperature profile $\theta(\eta)$ versus $\eta$ for set of values of $S, Q^{*}$ and 


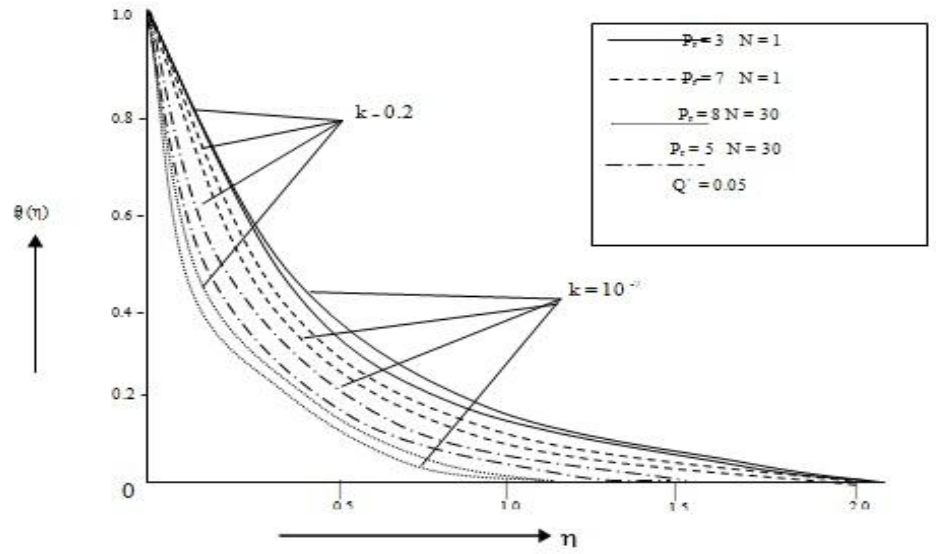

Fig. (4b) Dimensionless temperature profiles $\theta(\eta)$ for various values of Prandtl number $P_{r}$ and radiation parameter $\mathrm{N}$ when $\mathrm{V}_{0 x}=0.04$

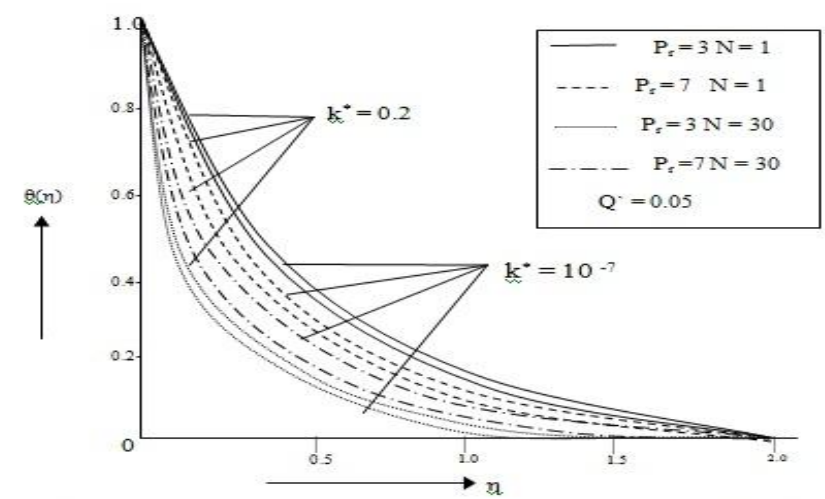

Fig, (4a) Dimensionless temperature profiles $\theta(\eta)$ for various values of Prandtl number $P_{\mathrm{r}}$ and radiation parameter $\mathrm{N}$ when $\mathrm{V}_{\mathrm{N}}=-0.04$

\section{Conclusions:}

The important conclusions of the study are as follows:

1. The horizontal velocity is maximum at $\eta=0$ for all values of $k_{2}, M$ and $S$. and decreases rapidly with increasing values of $\eta$.

2. The longitudinal velocity decreases with rise in the magnitude in the suction parameter $\mathrm{S}$.

3. The transverse velocity is minimum at $\eta=0$ for all values of $S, k_{2}$ and $M$ and increases slowly with increasing values of $\mathrm{S}, \mathrm{k}_{2}$ and $\mathrm{M}$.

4. An increase in suction parameter $\mathrm{S}$, wall temperature parameter $\mathrm{P}$ or heat sink parameter $\mathrm{Q}$ results in lowering the temperature field steadily.

5. An increase in the permeability parameter and magnetic parameter increases the skin-friction while an increase in suction parameter decreases the skin-friction.

6. The combined effect of increasing values of Prandtl number $\mathrm{P}_{\mathrm{r}}$ and radiation parameter $\mathrm{N}$ is to reduce the temperature largely in the boundary layer flow region.

7. The effect of the suction parameter $\mathrm{v}_{\mathrm{w}}$ is to increase the numerical value of wall temperature gradient and that of blowing is to decrease the same for all values of small visco-elastic parameter $k_{2}$, Prandtl number $P_{r}$ and radiation parameter $\mathrm{N}$ in absence of frictional heating. 


\section{References}

[1] A. Rapits, C. Perdikis, Viscoelastic Flow by the Presence of Radiation. ZAMM, 78 (1998), 277-279.

[2] A. Raptis, Technical note, Flow of a Micropolar Fluid Past a Continuously Moving Plate by the Presence of Radiation. Int. J. Heat Mass Transfer, 41 (1998.), 2865-2866.

[3] A. Raptis, Radiation and Viscoelastic Flow. Int. Comm. Heat Mass. Transfer, 26, (1999), 889-895.

[4] A. Raptis, C. Perdikis, H. S. Takhar, Effect of Thermal Radiation on MHD Flow. Appl. Math. Comput. 153 (2004), 645-649.

[5] Ajay Kumar Singh., Heat transfer and boundary layer flow past a stretching porous wall with temperature gradient dependent heat sink. J.E.H.M.T., 28 (2006), 109-125.

[6] Angirasa, D., Peterson, G.P., and Pop, I., Combined Heat Mass Transfer by Convection in a Saturated Thermally Stratified Porous Medium. Numerical Heat Transfer, A31 (1997), 255-271.

[7] Anjalidevi, S.P., and Thiyagarajan, M., Nonlinear Hydromagnetic Flow and Heat Transfer Over a Surface Stretching with a Power Law Velocity, Heat and Mass Transfer, 38 (2002), 723-726.

[8] B.S.Dandapat, A.S. Gupta, Flow and Heat Transfer in a Visco-elastic Fluid over a Stretching Sheet.Int. J. Non-Linear Mech, 24 (1989), 215-219.

[9] Hong, J. T., and Tien, C.L., Analysis of Thermal Dispersion, Effect on Vertical Plate Natural Convection in Porous Media. International Journal of Heat Mass Transfer, 30 (1987), 143-150.

[10] Khan, S.K., Abel, M.S., and Sonth, R.M., Visco-elastic MHD Flow Heat and Mass Transfer Over a Stretching Sheet with Dissipation of Energy and Stress Work. Heat and Mass Transfer, 40 (2004), 47-57.

[11] M. Kumari, G. Nath, Radiation Effect on Mixed Convection from a Horizontal Surface in a Porous Medium. Mech. Res. Comm., 31 (2004), 483-491.

[12] M.Q.Brewster, Thermal Radiative Transfer Properties. JhonWiky and Sons. (1972).

[13] M.S.Sarma, B.N. Rao, Heat Transfer in a viscoelastic fluid over a Stretching Sheet. J. Math. Anal. Appl. 222 (1998), $268-275$.

[14] McCormac, P.D., and Crane, L.J., Physical Fluid Dynamics. Academic Press, New York. (1973).

[15] R. Cortell, Flow and Heat Transfer of an Electrically Conducting Fluid of Second Grade over a Stretching Sheet Subject to Suction and to a transverse magnetic field. Int. Journal of Heat Mass Transfer, 49 (2006), 1851-1856.

[16] R. Cortell, A note on Flow and Heat Transfer of a Viscoelastic Fluid over a Stretching Sheet. Int. J. Non-Linear Mech., 41(2006), 78-85.

[17] Rafael Cortell Bataller, Viscoelastic Fluid Flow and Heat Transfer over a Stretching Sheet under the effects of a non-uniform Heat Source, Viscous Dissipation and Thermal Radiation.. Int. Journal of Heat and Mass Transfer, 50 (2007), 3152-3162.

[18] Rajagopal, K.R., Na, T.Y., and Gupta, A.S., Flow of a Viscoelastic Fluid Over a stretching Sheet. Rheol. Acta, 23 (1984), $213-221$.

[19] Sakiadis, B.C., Boundary Layer Behaviour on Continuous Solid Surfaces: I. Boundary Layer Equations for Two-dimensional and Axisymmetric Flow I.I.Ch.E.J., 7 (1961), 26-34.

[20] Schlichting, H., and Gersten, K., Boundary Layer Theory. Springer Verlag. 2000.

[21] Sujit Kumar Khan, Heat Transfer in a Visco-elastic Fluid Flow Over a Stretching Surface with Heat Source/Sink, Suction/Blowing and Radiation. Int. J.. Heat Mass Transfer, 49 (2006), 628-639.

[22] Surmadevi, C.D., Takhar, H.S., and Nath, G.,Unsteady Three-Dimensional Boundary Layer Flow Due to a Stretching Sheet. International Journal of Heat Mass Transfer, 29 (1986), 1996-1999.

[23] Takhar, H.S., Chamkha, A.J., and Nath, G., Natural Convection Flow from a Continuously Moving Vertical Surface Immersed in a Thermally stratified Medium. Heat and Mass Transfer, 38 (2001), 17-24.

[24] Takhar, H.S., and Soundalgekar. V.M., Flow and Heat Transfer of a Micropolar Fluid Past a Continuously Moving Porous Plate. International Journal of Engng. Sci., 23 (1985), 201-209.

[25] Vafai, K., and Tien, C. L. Boundary and Inertia Effects on Flow and Heat Transfer in Porous Media. International Journal of Heat Mass Transfer, 24 (1981), 195-203.

[26] Wang, C. Y. The three-Dimensional Flow Due to Stretching Flat Surface. Phys. Fluids, 27 (1984), 1915-1921. 